\title{
Los Restos Faunísticos de los Niveles Superiores de Cueva Los Carneros (Alto Chacabuco, XI Región, Chile)
}

\author{
Faunal Remains from the Upper Levels of Los Carneros Cave (Alto Chacabuco, XI \\ Region, Chile)
}

\author{
Francisco Mena Larraín ${ }^{\mathrm{i}}$ Patricio López Mendoza ${ }^{\mathrm{ii}}$
}

\begin{abstract}
RESUMEN
Se presentan los resultados del análisis zooarqueológico y tafonómico de los niveles superiores del sitio Cueva Los Carneros (CLC). El registro arqueofaunístico está conformado por una diversidad de taxa que representan el mosaico de microambientes asociados al sitio. En efecto, CLC corresponde a una localidad caracterizada por la caza diversificada, en la cual las especies con mayor frecuencia de captura y consumo corresponden a variados roedores, Lama guanicoe, Zaedyus pichiy, Rhea pennata, Hippocamelus bisulcus, aves, entre otros, los que se unen a otros taxa (como roedores y lagartijas) depositados mediante causas naturales y antrópicas dentro de una dinámica esperable para refugios rocosos. Las evidencias arqueofaunísticas no permiten plantear con certeza un uso estival, mientras que la frecuencia de restos de guanacos, huemules y otros taxa sugieren explotaciones orientadas al consumo de grasas-médulas y un traslado de otras unidades dentro una lógica de movilidad pautada.
\end{abstract}

Palabras Clave:Alto Chacabuco, Cueva, Estepa, Zooarqueología, Tafonomía

\begin{abstract}
The results of the zooarchaeological and taphonomical analysis of the materials recovered at the upper levels from Cueva Los Carneros are presented. The archaeofaunal record is shaped by a diversity of taxa that represent the mosaic of microenvironments around the site. In effect, CLC corresponds to a locality characterized by diversified hunt, in which the species with major frequency of capture and consumption correspond to several rodents, Lama guanicoe, Zaedyus pichiy, Rhea pennata, Hippocamelus bisulcus and birds, among others. These are joined by many taxa (like rodents and lizards) that were deposited by natural and antrophic agents integral to the dynamics of all rock shelters. Although archaeofaunal data do not clearly point to a summertime occupation, whereas the frequency of gunanacos, huemules and other taxa suggest hunting activities geared towards grease-
\end{abstract}

i Centro de Investigación en Ecosistemas de la Patagonia (CIEP; CONICYT-Regional R10C 1003) Bilbao 323 of. 216, Coyhaique, Chile. Correo-e: francisco.mena@ciep.cl

ii Programa de Doctorado en Antropología, Universidad Católica del Norte, Instituto de Investigaciones Arqueológicas y Museo Gustavo Le Paige (IIAM), Calle Gustavo Le Paige No 380, San Pedro de Atacama, Chile. Correo-e: patriciolopezmend@yahoo.es

Recibido: 12-12-2012 Revisado: 03-01-2014 Aceptado: 10-05-2014 
medulla consumption and the transport of ther anatomical units within a patterned mobility strategy.

Key Words:Alto Chacabuco, Cave, Steppe, Zooarchaeology, Taphonomy

\section{INTRODUCCIÓN}

Diversas prospecciones han confirmado que Cueva Los Carneros (CLC en adelante) y Alero Entrada Baker son los únicos dos reparos rocosos susceptibles de haber albergado a un grupo de unas 4 a 5 personas en esta zona superior del Río Chacabuco o Entrada Baker (Mena y Lucero 2004, Goñi et al. 2004, Reyes et al. 2006). El segundo de estos sitios ha sido bastante estudiado y es bien conocido en la literatura, siendo el gran referente para elaborar hipótesis para la interpretación de la prehistoria de todo este territorio (Mena y Jackson I99I, Méndez y Velásquez 2005). De acuerdo a esto, era relevante estudiar CLC puesto que si bien no presentaba evidencias arqueológicas superficiales de ningún tipo, un sondeo practicado en el sitio en el año 2000 reveló restos indudables de ocupación indígena. La oportunidad de emprender esta tarea se dio en el marco de un proyecto de turismo científico (PTC-2) coordinado por el Centro de Investigación en Ecosistemas de la Patagonia (CIEP) con el financiamiento de INNOVA-Corfo y en enero de 201 I se practicaron nuevas excavaciones en el sitio, recuperando la colección ósea que se informa ahora.

CLC (279887 E - 477254I N; 600 msnm) es una cueva estructural de aproximadamente $10 \mathrm{~m} \chi 15$ $m$ excavada en las paredes ignimbríticas de un pequeño cañadón por el que corre uno de varios arroyuelos que finalmente vierten sus aguas a un naciente Río Chacabuco (ver Figura la y b). Aunque el área circundante está dominada por un paisaje de estepa graminosa, este cuerpo de agua inmediato, así como otros más lejanos, una importante laguna permanente sobre el sitio o la cercana presencia de bosques de falda y estepa altoandina, constituyen un mosaico de microambientes susceptibles de haber sido explotados. Uno de los principales objetivos de esta reexcavación era evaluar varias hipótesis basadas en los estudios previos en Alero Entrada Baker. Una fecha radiocarbónica en la Capa 2B dio I.285 \pm 25 AP (UGAMS 879I), lo que indica que un supuesto hiato de abandono entre 6.500 y $300 \mathrm{AP}$ no es tal (ver Figura IB). Específicamente, el presente estudio apunta a ver si todos los recursos disponibles fueron explotados o bien provienen de depositaciones naturales y si además, la estructura de edad y otros atributos contribuyen a responder preguntas relativas a las estaciones de ocupación del sitio y a las actividades en éste desarrolladas. Por último, se buscó evaluar la hipótesis de que hacia momentos más tardíos la movilidad debió estar más pautada en circuitos logísticos.

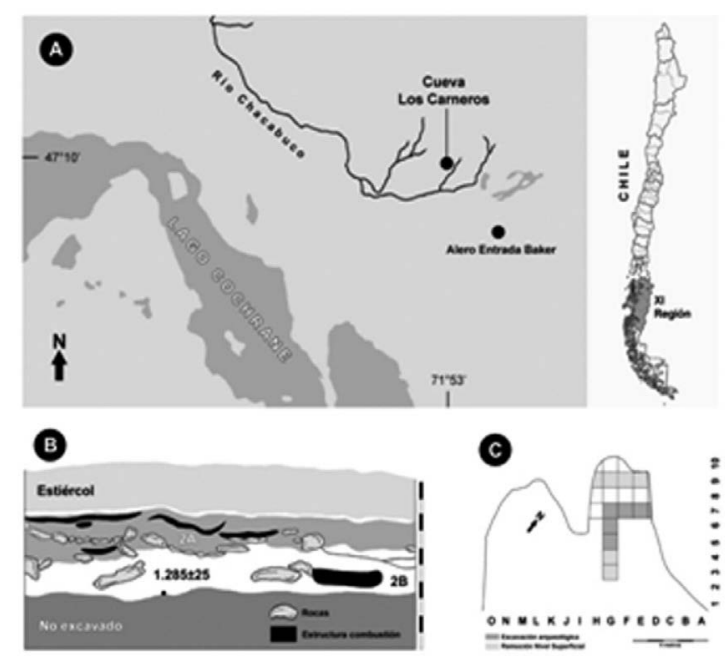

Figura 1: a) Ubicación del sitio, b) Perfil estratigráfico de la Cueva Los Carneros y c) Planta del sitio.

Figure 1: a) Location of the site, b) Stratigraphic profile of Cueva Los Carneros and c) Plant view of the site.

\section{MATERIAL Y MÉTODOS}

Un total de 5.859 especímenes provenientes del sitio CLC fueron analizados. De la Capa I provienen 2 restos óseos asignados a mamíferos indeterminados, por lo que el presente análisis se enfocó en el material osteofaunístico de la Capa 2. Todo este registro proviene de la unidades $2 \mathrm{~F}, 5 \mathrm{G}, 6 \mathrm{C}$, 6G, 7D, 7F y 7G que se distribuyeron al centro de la cueva en la cámara que da hacia el NE (ver Figura Ic). EI énfasis del análisis zooarqueológico y tafonómico, está en generar un panorama amplio de las alteraciones tanto culturales como naturales del registro, así como identificar tendencias en los rangos etáreos de los principales taxa para así evaluar el uso estacional del sitio y la hipótesis de que hacia momentos más tardíos la movilidad debió estar más pautada. 
La abundancia de los diferentes taxa, fue cuantificada por medio del NISP y MNI, mientras que la abundancia anatómica de cada taxa se calculó mediante el MNE, MAU y \%MAU. Se evaluó además para el caso del guanaco el $M N E_{\text {observado }}$ versus el $M N E_{\text {es: }}$ con el fin de evaluar la predominancia de secciones de la anatomía animal por sobre otras (ver De Nigris 2004). Para evaluar posibles efectos de preservación diferencial en la muestra de guanaco se correlacionaron valores de densidad ósea (DO) (Stahl 1999) y el \%MAU considerando las muestras sin separación de niveles, tomando la Capa 2 como un mismo bloque. Para evaluar la frecuencia de los restos de guanacos en relación a su utilidad económica se realizó el mismo ejercicio con el \%MAU y los valores de utilidad (IU) de Borrero (1990, modificados por Lyman 1994), utilidad de la cavidad medular (CM) de huesos largos (Mengoni Goñalons 1996) e índices de secado (IS) (De Nigris y Mengoni Goñalons 2004). Para la estimación de los perfiles de edad se utilizaron las muestras de Hippocamelus bisulcus y Lama guanicoe de la Capa 2. Este análisis se desarrolló de acuerdo a los criterios propuestos por Mena (1992) y Kauffman (2004).

\section{RESULTADOS}

El registro osteofaunístico de Cueva Los Carneros (CLC) comprende una gran diversidad de taxa de mamíferos, aves, anfibios y reptiles (ver Figura 2a-d y Tabla I). Esta diversidad faunística refleja los variados ambientes aledaños al sitio como la estepa graminosa, cuerpos de aguas, bosque de falda y estepa altoandina. La presencia de Ovis aries en Capa 2 y hasta el Nivel IV $(30-40 \mathrm{~cm})$ no indica únicamente que el refugio fue ocupado en tiempos históricos, sino también la migración por la acción de animales fosoriales que alteraron en parte la estratigrafía del sitio. Este tipo de alteraciones es común en Patagonia central y ha sido previamente abordadas y discutidas (ver López y Mena 20II). A esto se debe agregar la incorporación de fecas dejadas por carnívoros a decir por los rastros de ácidos digestivos en 256 especímenes entre los que destacan restos de Lama guanicoe, Rhea pennata y roedores varios, además de egagrópilas de aves rapaces que habitan ocasionalmente los roqueríos que dieron forma al refugio (ver Figura 3a, b).
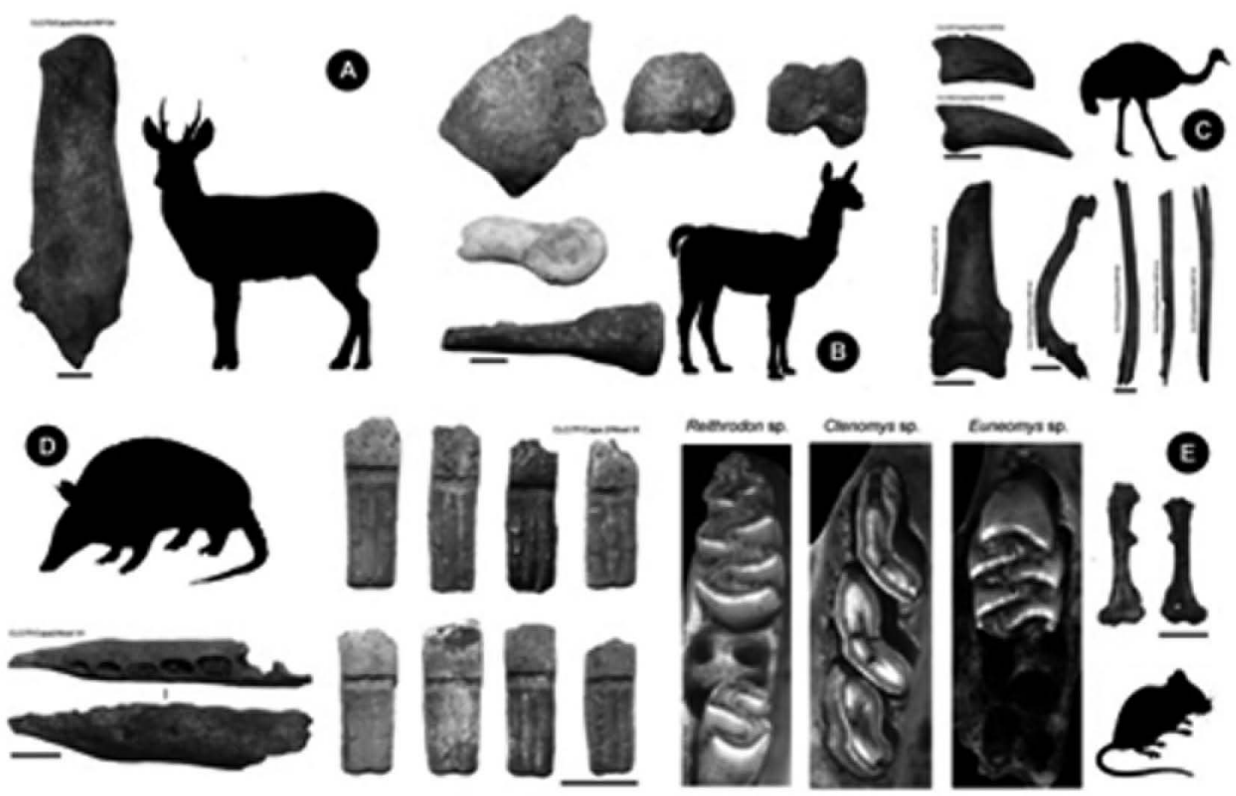

Figura 2: Registros faunístico del sitio Cueva Los Carneros, a) restos de Hippocamelus bisulcus, b) Lama guanicoe, c) Rhea pennata, d) Zaedyus pichiy, y e) roedores varios. Escalas $=1 \mathrm{~cm}$.

Figure 2. Faunal remains of Cueva Los Carneros, a) Hippocameluds bisulcus remains, b) Lama guanicoe, c) Rhea pennata, d) Zaedyus pichiy, ye) different rodents. Scales $=1 \mathrm{~cm}$. 
De acuerdo al análisis en huesos largos y dientes se observa para el caso de los guanacos una predominancia de restos fusionados $(\mathrm{MNE}=138)$ por sobre los no fusionados ( $M N E=44)$, aunque se identificó una mayor abundancia de dientes deciduos $(M N E=10)$ por sobre los permanentes $(M N E=4)$. Sobre esto último, cabe mencionar que los dientes deciduos suelen convivir durante un tiempo con los molares permanentes, por lo que el registro aislado de estos especímenes no es del todo diagnóstico para discutir las edades de los individuos.
En un total de 122 elementos no fue posible observar el estado de fusión. Dentro de los restos no fusionados, los mismos son asignables a animales juveniles que van de I a 2 años. Una situación similar a la de los restos de Lama guanicoe se observa en el material de Hippocamelus bisulcus ya que dominan los huesos fusionados (MNE $=9$ ) por sobre los no fusionados (MNE = 3), mientras que únicamente 3 dientes permanentes fueron registrados por sobre I diente deciduo (véase Tabla 2).

\begin{tabular}{|c|c|c|c|c|c|c|c|c|c|}
\hline \multirow[b]{2}{*}{ Taxa } & \multicolumn{4}{|c|}{ Capa I } & \multicolumn{5}{|c|}{ Capa 2} \\
\hline & II & III & III-IV & IV & $\mathbf{v}$ & VI & VII & VIII & IX \\
\hline Anura & 0 & 0 & 0 & 2 & 1 & 4 & 9 & I & I \\
\hline Lacertilia & 0 & 0 & 0 & 0 & 2 & 0 & 0 & 0 & I \\
\hline Liolaemus sp. & $0(0)$ & $0(0)$ & $0(0)$ & $16(4)$ & $3(2)$ & $42(I I)$ & $17(6)$ & $0(0)$ & $0(0)$ \\
\hline Artiodactyla & 0 & 1 & 12 & 20 & 8 & 18 & 4 & 0 & 0 \\
\hline Lama guanicoe & $0(0)$ & $I(I)$ & $5(1)$ & $78(5)$ & $60(3)$ & $55(4)$ & $60(3)$ & $54(3)$ & $7(I)$ \\
\hline Hippocamelus bisulcus & $0(0)$ & $0(0)$ & $5(2)$ & $3(2)$ & $8(2)$ & $0(0)$ & $I(I)$ & $0(0)$ & $0(0)$ \\
\hline Ovis aries & $0(0)$ & $0(0)$ & $6(1)$ & $67(4)$ & $0(0)$ & $I(I)$ & $0(0)$ & $0(0)$ & $0(0)$ \\
\hline Ave & 0 & 0 & 0 & 3 & 13 & 6 & 5 & 3 & 1 \\
\hline Passeriforme & 0 & 0 & 0 & 3 & $\mathrm{I}$ & 2 & 0 & 0 & 0 \\
\hline Rhea pennata & $0(0)$ & $0(0)$ & $I(I)$ & $6(1)$ & $15(I)$ & $0(0)$ & $2(I)$ & $I(I)$ & 0 \\
\hline Carnivora & 0 & 0 & 0 & 37 & I & 0 & 0 & I & 0 \\
\hline Canidae & 0 & 0 & 0 & I & $\mathrm{I}$ & 0 & 0 & 0 & 0 \\
\hline Felidae & 0 & 0 & 0 & 0 & I & 2 & 0 & 0 & 0 \\
\hline Lycalopex culpaeus & $0(0)$ & $0(0)$ & $\mathrm{I}(\mathrm{I})$ & $5(2)$ & $0(0)$ & $I(I)$ & $I(I)$ & $3(1)$ & $0(0)$ \\
\hline Puma concolor & $0(0)$ & $0(0)$ & $0(0)$ & $2(1)$ & $0(0)$ & $0(0)$ & $0(0)$ & $0(0)$ & $0(0)$ \\
\hline Rodentia & 0 & 0 & 25 & 654 & 725 & 734 & 329 & 235 & 81 \\
\hline Ctenomys sp. & 0 & 0 & $I(I)$ & III (26) & $52(17)$ & $142(38)$ & $68(17)$ & $57(20)$ & $17(5)$ \\
\hline Euneomys sp. & $0(0)$ & $0(0)$ & $0(0)$ & $\mathrm{I}(\mathrm{I})$ & $12(5)$ & $3(2)$ & $I(I)$ & $2(1)$ & $0(0)$ \\
\hline Loxodontomys sp. & $0(0)$ & $0(0)$ & $0(0)$ & $4(3)$ & $2(1)$ & $2(I)$ & $0(0)$ & $2(1)$ & (0) \\
\hline Microcavia australis & $0(0)$ & $0(0)$ & $0(0)$ & $0(0)$ & $\mathrm{I}(\mathrm{I})$ & $0(0)$ & $0(0)$ & $0(0)$ & $0(0)$ \\
\hline Phyllotis sp. & $0(0)$ & $0(0)$ & $I(I)$ & $34(10)$ & $24(9)$ & $21(8)$ & $0(0)$ & $5(2)$ & $4(2)$ \\
\hline Reithrodon sp. & $0(0)$ & $0(0)$ & $I(I)$ & $36(15)$ & $65(28)$ & $36(13)$ & $29(13)$ & $6(2)$ & $3(2)$ \\
\hline Zaedyus pichiy & $0(0)$ & $0(0)$ & I (I) & $10(1)$ & $8(1)$ & $18(1)$ & $11(1)$ & $0(0)$ & $40(1)$ \\
\hline Indeterminado & 0 & 0 & 0 & 3 & 2 & 3 & I & 0 & 0 \\
\hline Mammalia & 2 & 0 & 96 & 448 & 206 & 277 & 178 & 286 & 51 \\
\hline TOTAL & 2 & 2 & 155 & 1544 & $|2|$ | & 1367 & 716 & 656 & 206 \\
\hline
\end{tabular}

Tabla 1: Taxa identificados en el sitio Cueva Los Carneros expresados en NISP y MNI (entre paréntesis), separados por Capa estratigráfica y Nivel de excavación. Para el cálculo de MNI únicamente se utilizaron los taxa identificados a nivel de género y/o especie.

Table 1: Taxa identified in the site Cueva Los Carneros expressed in NISP and MNI (in brackets), separated by Stratigraphic layer and Level of excavation. 


\begin{tabular}{|c|r|r|r|r|r|r|r|r|r|r|r|}
\hline & \multicolumn{2}{|c|}{ Total } & \multicolumn{2}{|c|}{ Fusionados } & \multicolumn{2}{c|}{$\begin{array}{c}\text { No } \\
\text { Fusionados }\end{array}$} & \multicolumn{2}{c|}{$\begin{array}{c}\text { Fusión } \\
\text { Indeterminada }\end{array}$} & \multicolumn{2}{c|}{$\begin{array}{c}\text { Dientes } \\
\text { permanentes }\end{array}$} & \multicolumn{2}{c|}{$\begin{array}{c}\text { Dientes } \\
\text { deciduos }\end{array}$} \\
\hline \multicolumn{1}{|c|}{ TAXA } & MNE & \multicolumn{1}{|c|}{ MNE } & \multicolumn{1}{c|}{$\%$} & MNE & \multicolumn{1}{c|}{$\%$} & \multicolumn{1}{c|}{ MNE } & \multicolumn{1}{c|}{$\%$} & MNE & $\%$ & MNE & $\%$ \\
\hline Lama guanicoe & 318 & 138 & 43.4 & 44 & 13.8 & 122 & 38.4 & 4 & 1.3 & 10 & 3.1 \\
\hline Hippocamelus bisulcus & 17 & 9 & 52.9 & 3 & 17.6 & 0 & 0 & 4 & 23.5 & 1 & 5.9 \\
\hline
\end{tabular}

Tabla 2: Estructura etárea de los especímenes de Lama guanicoe e Hippocamelus bisulcus expresados en MNE y \%MNE.

Table 2: Age strcture of the specimens of Lama guanicoe and Hippocamelus bisulcus expressed in MNE and \%MNE.
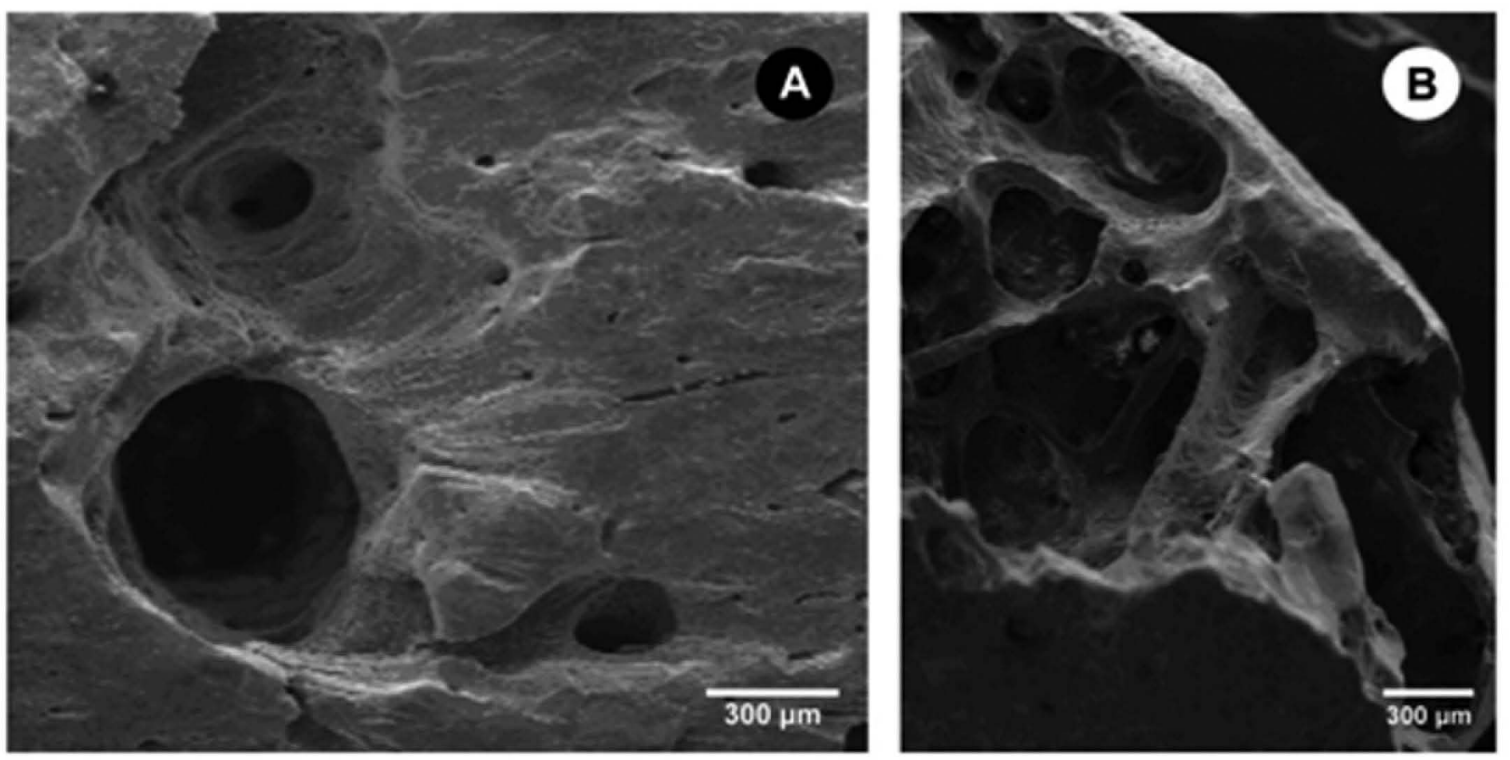

Figura 3: Rastros de ácidos digestivos en A. sesamoideo de Lama guanicoe y B. húmero de Cricetidae.

Figure 3: Marks of digestive acids in A. Lama guanicoe sesamoid and B. Cricetidae humerus.

\section{Abundancia y frecuencia anatómica}

Dentro del conjunto de guanacos adultos de la Capa 2 se observa una predominancia del cráneo y extremidades superiores y medias por sobre el resto de unidades del esqueleto apendicular y axial (véase Figura 4a). Por su parte, los guanacos juveniles son más escasos, y las unidades de las extremidades inferiores son las más representadas (véase Figura $4 b$ ). Tanto las extremidades superiores y medias poseen cantidades de médula altas a moderadas, mientras que el cráneo aporta grasa. Unidades como costillas y vértebras se encuentran escasamente representadas tanto para los guanacos adultos como juveniles (véase Figura $4 \mathrm{a}$ y $4 \mathrm{~b}$ ). El resultado del análisis de correlaciones para el caso de DO en animales adultos es negativo con una baja significancia estadística, misma situación que se observa para las correlaciones del IU (véase Tabla 3). Para el caso de la cavidad medular en huesos largos el resultado de la correlación es positivo y con una baja significancia estadística. Estas unidades no se encuentran afectadas mayormente por la acción de carnívoros puesto que un $8.8 \%$ de la totalidad de los restos de guanacos presentaban signos asociados a este tipo de daños, aspecto esperable para la baja capacidad de carroñeros en Patagonia para destruir huesos (Fernández 2008). Lo anterior sostiene que la representación ósea de guanacos en CLC se explica más bien por tendencias en el procesamiento y consumo de unidades con altos a medios niveles de grasas y médula. 

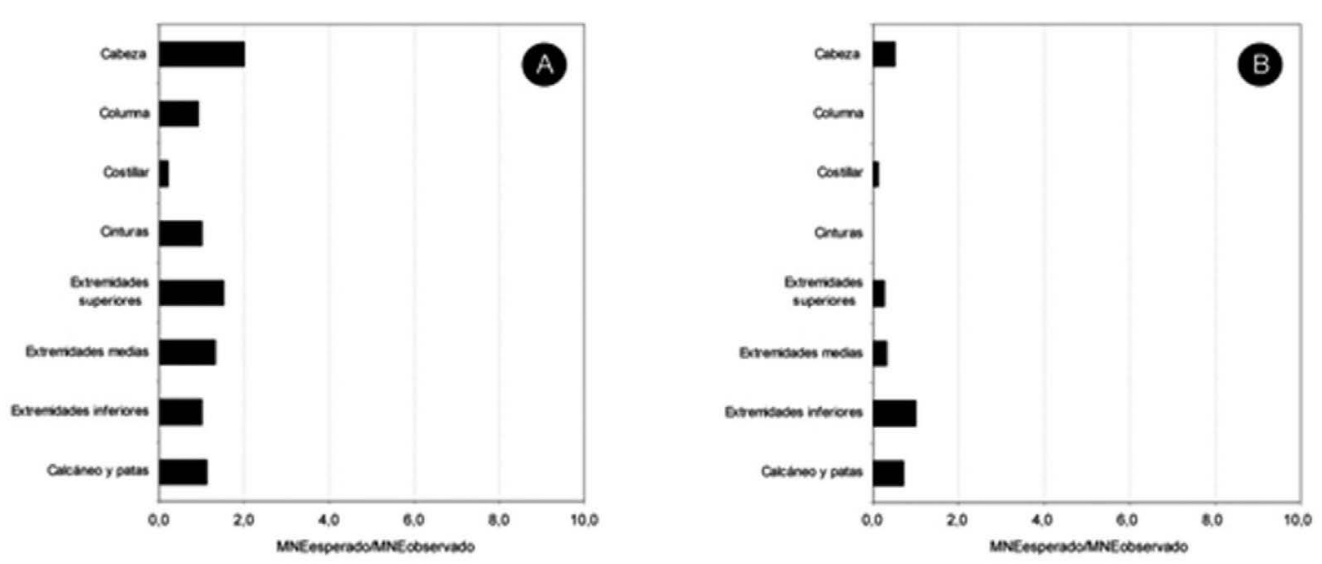

Figura 4: Tendencias en la representación de unidades anatómicas de guanacos, A. adultos, B. juveniles.

Figure 4: Trends in the representation of guanacos anatomical units, A. adults, B. juveniles.

\begin{tabular}{|l|c|c|}
\hline \multicolumn{3}{|c|}{ Lama guanicoe } \\
\hline \%MAU-DO* & $r_{s}=-0.016$ & $P=0.928$ \\
\hline \%MU-IU ${ }^{* *}$ & $r=-0.084$ & $P=0.632$ \\
\hline$\% M A U-\mathrm{CM}^{+}$ & $r_{s}=0.308$ & $P=0.502$ \\
\hline \%MUU-IS $^{++}$esqueleto axial & $r=-0.655$ & $P=0.158$ \\
\hline \%MAU-IS $^{++}{ }_{\text {esqueleto apendicular }}$ & $r=-0.266$ & $P=0.458$ \\
\hline
\end{tabular}

Tabla 3: Coeficientes de correlación (Pearson y Spearman) entre el \%MAU de camélidos adultos con valores de densidad ósea, utilidad económica (IU), cavidad medular de huesos largos (CM) (húmeros, radioulnas, fémures, tibias, metapodios y primeras falanges) e índice

de secado (IS) (esqueletos apendicular y axial). ${ }^{*}$ Densidad ósea, "Índice de utilidad, ${ }^{+}$Cavidad medular, ${ }^{++}$Índice de secado de carne

Table 3: Coefficients of correlation (Pearson and Spearman) between \%MAU of adult camelids with values of bony density, economic usefulness (IU), medular cavity of long bones (CM) (humerus, radius-ulna, femurs, tibias, metapodials and the first phalanxes) and index of dried (IS) (skeletons appendicular and axial). ${ }^{*}$ Bone density, ${ }^{* *}$ Dry Index, ${ }^{+}$Medullary cavity, ${ }^{++}$Meat Drying Index.

En cuanto a los resultados para el IS, tanto el esqueleto apendicular como axial los valores son negativos pero de baja significancia estadística. La baja frecuencia de secciones como la parrilla costal y de las cinturas tanto escapular como pélvica sugiere un traslado de carne a otros sitios. Los restos de

huemul son escasos y representan secciones del cráneo-mandíbula, extremidades superiores e inferiores, además de un calcáneo (Tabla 4). Esta representación es similar a la observada para el caso de los guanacos y puede responder a la misma estrategia de consumo.

\begin{tabular}{|l|c|c|c|c|}
\hline & \multicolumn{2}{|c|}{ Adultos } & \multicolumn{2}{c|}{ Juveniles } \\
\hline Elemento óseo & NISP & MNE & NISP & MNE \\
\hline Maxila & 2 & I & 0 & 0 \\
\hline Mandíbula & I & I & I & I \\
\hline Carpianos & 7 & 7 & 0 & 0 \\
\hline Fémur px & 0 & 0 & 2 & 2 \\
\hline Calcáneo & I & I & 0 & 0 \\
\hline Metapodio ds & I & I & 0 & 0 \\
\hline
\end{tabular}

Tabla 4: Representación anatómica de Hippocamelus bisulcus (juveniles y adultos) del sitio CLC. Table 4: Anatomical representation of Hippocamelus bisulcus (juvenile and adult) of the site CLC. 


\begin{tabular}{|l|c|c|}
\hline Elemento óseo & NISP & MNE \\
\hline Cervical & $\mathrm{I}$ & $\mathrm{I}$ \\
\hline Costilla & 8 & 7 \\
\hline Escápula & $\mathrm{I}$ & $\mathrm{I}$ \\
\hline Húmero & 3 & 3 \\
\hline Tibiotarso & $\mathrm{I}$ & $\mathrm{I}$ \\
\hline I falange & $\mathrm{I}$ & $\mathrm{I}$ \\
\hline II falange & 3 & 3 \\
\hline Falange ungueal & 7 & 7 \\
\hline
\end{tabular}

Tabla 5: Representación anatómica de Rhea pennata del sitio CLC. Table 5: Anatomical representation of Rhea pennata of the site CLC.

Los restos de Rhea pennata representan a un individuo y predominan restos de costillas, falanges ungueales, $y$ huesos largos, y la baja frecuencia de "choique" en el sitio indica un uso más bien esporádico de este recurso propio de la estepa (Tabla 5). Ahora bien, un total de 515 especímenes presentan signos de exposición al fuego $(\% \mathrm{NISP}=8.8$ en relación al total) de las cuales 28 corresponden a placas dérmicas de Zaedyus pichiy. La totalidad de los huesos largos presentan fracturas y en general se observó una baja representación de marcas de procesamiento concentradas en muestras de Lama guanicoe, Hippocamelus bisulcus, Felidae y Rhea pennata y asociadas a actividades de desarticulación $y$ fileteo.

\section{DISCUSIÓNY CONCLUSIONES}

El conjunto de las evidencias arqueofaunísticas del sitio CLC apuntan a varios aspectos relacionados a las hipótesis, objetivos y problemas antes mencionadas. En primer lugar, la diversidad de especies presentes indica un aprovechamiento extendido de todos los microambientes del sitio y de las zonas aledañas a éste. Las principales presas consumidas corresponden a Lama guanicoe e Hippocamelus bisulcus, seguidos por Zaedyus pichiy y Rhea pennata y roedores. En cuanto a las edades de los especimenes de Lama guanicoe e Hippocamelus bisulcus dominan los individuos adultos, y la baja frecuencia de individuos juveniles no permite sustentar esta hipótesis de ocupaciones estivales ya que en comparación al sitio de Alero Entrada Baker la abundancia de neonatos/ juveniles es menor en CLC (Mena y Jackson 199I), asociado además a la baja frecuencia de aves. La laguna cercana al sitio durante primavera-verano sustenta una gran diversidad y abundancia de aves, lo que no se traduce en el registro zooarqueológico. En el caso de los géneros de roedores de CLC, en su totalidad se reproducen entre un rango que va de Noviembre a Marzo (Figueroa et al. 200I), lo que es interesante para la muestra del sitio ya que abundan individuos juveniles de cricétidos y Ctenomyidae. No obstante, estos datos no permiten apoyar la hipótesis de estacionalidad por cuanto parte de este registro ingresó al sitio por vías naturales. Para el caso del huemul es frecuente encontrarlo en las épocas favorables entre los 700 y 900 msnm dentro de formaciones vegetacionales de bosque caducifolio de Aisén, bosque siempreverde mixto del Baker y a matorrales periglaciales (Muñoz y Yáñez 2009). No obstante, la ubicación de CLC no es un buen indicador de estacionalidad debido a los bosques de falda cercanos al sitio y a la diversidad de microambientes asociados.

La frecuencia de restos óseos, el procesamiento de cráneos y sobre todo huesos largos de guanacos y huemules, $y$ en menor medida de choique (Rhea pennata), indica un consumo intensivo de médulas y grasas, dentro de una fase inicial en el procesamiento de carcasas (Binford 1978). A esto se une la ausencia de secciones con aportes cárnicos que posiblemente fueron trasladadas dentro de una dinámica de movilidad más pautada en circuitos logísticos. La baja frecuencia de restos líticos y la presencia de artefactos óseos para el reavivado de filos en CLC indican además asentamientos poco prolongados pero intensivos. Por lo mismo, parte de la hipótesis preliminarmente postuladas para el sitio han logrado ser contrastadas; sin embargo, un tema a abordar en futuras excavaciones $y$ análisis son los indicadores de estacionalidad y el grado de visibilidad que éstos tienen a partir de una muestra zooarqueológica como la estudiada hasta la fecha. Así, surgen preguntas relacionadas a la baja frecuencia de aves y enfatizar otros registros que aún se encuentran en proceso de análisis como la evidencia lítica y arqueobotánica.

Agradecimientos: El presente trabajo fue llevado a cabo en el marco del proyecto "Las Ciencias al servicio del desarrollo turístico de Aysén, creando un Centro de Turismo Científico", coordinado por el CIEP y financiado por INNOVACorfo. 


\section{BIBLIOGRAFÍA}

Binford, L. 1978. Nunamiut Ethnoarchaeology. Academic Press. Nueva York.

Borrero, L. A. 1990. “Fuego-Patagonian bone assemblages and the problem of communal guanaco hunting". En Hunters of the recent past, editado por L. B. Davis y B. Reeves, Pp. 373-399. Unwin Hyman, Londres.

De Nigris, M. 2004. El Consumo en Grupos Cazadores Recolectores. Un Ejemplo Zooarqueológicos de Patagonia Meridional. Sociedad Argentina de Antropología, Buenos Aires.

De Nigris, M. y G. Mengoni Goñalons. 2004. “El guanaco como fuente de carne y grasas en Patagonia”. En Contro viento y marea. Arqueología de Patagonia, editado por M.T. Civalero P. M. Fernández y A. G. Guráieb, pp. 469-476. Sociedad Argentina de Antropología y Instituto Nacional de Antropología y Pensamiento Latinoamericano, Buenos Aires.

Fernández, P. 2008. "Taphonomy and zooarchaeology in the Neotropics:A view from Northwestern Patagonian forest and steppe”. Quaternary International 180: 63-74.

Figueroa, R., S. Corales, J. Cerda y H. Saldivia. 200I. Roedores, Rapaces y Carnívoros de Aysén. Servicio Agrícola y Ganadero, Gobierno Regional de Aysén, Coyhaique, Chile.

Goñi, R., G. Barrientos, M. J. Figuerero, G. Mengoni, F. Mena, V. Lucero y O. Reyes. 2004. "Distribución espacial de entierros en la cordillera de Patagonia centro-meridional (Lago Salitroso-Paso Roballos, Argentina / Entrada Baker-Chacabuco, Chile)". Chungará 2 (Volumen Especial): I I0I-II07.

Kaufmann, C. 2004. La fusión ósea como indicador de edad y estacionalidad en guanaco (Lama guanicoe). En Contra Viento y Marea:Actas de lasV Jornadas de Arqueología de la Patagonia, editado por T. Civalero, G. Guraieb y P. Fernández, pp. 477-487. Instituto Nacional de Antropología y Pensamiento Latinoamericano y Sociedad Argentina de Antropología, Buenos Aires.

López, P. y F. Mena. 20I I. “Extinct ground sloth dermal bones and their role in the taphonomic research of caves: the case of Bano Nuevo-I (Andean Central Patagonia, Chile)." Revista Mexicana de Ciencias Geológicas 27(2): 519-532.
Lyman, R. L. 1994. Vertebrate Taphonomy. Cambridge Manuals in Archaeology. Cambridge University Press, Cambridge.

Mena, F. 1992. "Mandíbulas y maxilares: un primer acercamiento a los conjuntos arqueofaunísticos del Alero Fontana”. Boletín del Museo Nacional de Historia Natural 43: I79I9I.

Mena, F. y D. Jackson. 1991. "Tecnología y subsistencia en Alero Entrada Baker, Región de Aisén”. Anales del Instituto de la Patagonia Ser. Cs. Soc. 20: 169-203.

Mena, F. y V. Lucero. 2004. "En torno a las últimas poblaciones indígenas de la cordillera centro-patagónica. Estudio comparado de tres valles en Aisén oriental (Chile)". En Contra Viento y Marea: Actas de las V Jornadas de Arqueología de la Patagonia, editado por T. Civalero, G. Guraieb y P. Fernández, pp. 643-658. Instituto Nacional de Antropología y Pensamiento Latinoamericano y Sociedad Argentina de Antropología, Buenos Aires.

Méndez., C. y H. Velásquez. 2005. "Tecnología y subsistencia en Alero Entrada Baker: una revisión a la luz de nuevos antecedentes". Actas XVI Congreso Nacional de Arqueología Chilena pp. 593-602. Tomé.

Mengoni-Goñalons, G. 1996. “La domesticación de los camélidos sudamericanos y su anatomía económica”. En Zooarqueología de Camélidos 2. Grupo Zooarqueología de Camélidos Perspectivas Teóricas y Metodológicas 2, editado por D. Elkin, C. Madero, G. Mengoni-Goñalons, D. Olivera, M. Reigadas y $\mathrm{H}$. Yacobaccio, pp. 3-45, GZC, Buenos Aires.

Muñoz, A. y J. Yáñez. 2009. Mamíferos de Chile. Cea Ediciones, Valdivia.

Reyes, O., C. Méndez, H. Velásquez y V. Trejo. 2006. Distribuciones espaciales y contexto de cazadores recolectores esteparios en el Alto Río Cisnes (XI Región de Aisén). Magallania 24(2): 75-90.

Stahl, P. 1999. "Structural Density of Domesticated South American Camelid Skeletal Elements and the Archaeological Investigation of Prehistoric Andean Ch'arki". Journal of Archaeological Science 26: I347-1368. 